\title{
Optimal Tone-Mapping for HDR Images
}

\author{
N. Neelima, Y. Ravi Kumar
}

\begin{abstract}
This research paper proposes a unique optimal tone-mapping technique for high dynamic range (HDR) images, performing local adjustments with overlapping windows covering complete image. A local linear adjustment is applied on each window to preserve the radiance values. This problem may be treated as global optimization problems to satisfy the local restriction for every overlapping window. These Local constraints may be considered as a guidance map to suppress high contrast without losing its details. M-estimation technique may be used for solving this optimization problem. This technique may be applied to HDR images with sudden radiance changes or comparatively smooth transitions. Further, this technique may be applied to differentiate and analyzes $H D R$ images from LDR images. Simulation results are included to support the performance gains achieved by the proposed technique.
\end{abstract}

Keywords- HDR, M-estimation, image compression, low dynamic range tone mapping.

\section{INTRODUCTION}

In recent years, tone mapping techniques have received a great deal of attention [1]. A high dynamic range (HDR) method consists of local and global tone mapping [2]. The Global tone mapping is done through the operator map with radiance values in spatially invariant manner by establishing a one- by one high dynamic radiance value to the low dynamic value. Non-linear global operators were proposed in [3]; in which global tone reproduction curves for many parameters of images are predicted to minimize the dynamic range by using computational methods. But, these techniques may be unsuccessful for HDR images as local regions contain values of highier and lower radiances. Further, fitting a relevant tone reproduction curve is very crucial for several cases. Local operators involve decomposing, compressing, and recombining layers in the HDR images [4]. In [5], the authors have proposed an edge preserving multi-scale image decomposition technique. An associated method to adjust the tonal values locally was represented in [6] and influence functions are also computed to restrict the updated modifications of tonal value in image. Detecting the secular highlights along with boosting the intensity in the regions of LDR images was proposed in [7]. Optimal clustering for detection and cluster center initialization algorithm along with effective tone mapping was proposed by the authors in [8]. Cluster centers were calculated using density based multi level DSA(Data Suppression Algorithm).

Hence, a new influence function [9-10] for local tone mapping operator is designed in this research work which doesn't depends on segmentation or multiscale

Revised Version Manuscript Received on 10, September 2019.

N. Neelima, Associate Professor, ECE Department, CMR Institute of Technology, Hyderabad, Telangana, India(E-Mail neelu.nimmagadda@gmail.com)

Y. Ravi Kumar, SC-G, DLRL, Hyderabad, Telangana, India.(E-mail : dr.ravikumaryeda@gmail.com) decomposition of HDR images into fractional maps to abstain the problems related with layer decomposition. The

radiance map is directly processed for developing global optimization framework to constrict the pixel values in a global sense. Further, smoothness impulsions to be inflicted with eventual output are not required in the proposed approach.

Further the research work is clustered as follows: Section 2 illustrates proposed algorithm for HDR images. Section 3 presents HDR image enhancement and synthesis. Simulation results are presented in Section 4 followed by concluding remarks.

\section{CONSTRUCTING THE HIGH DYNAMIC RANGER RADIANCE MAP}

Accustomed a HDR input image with radiance map $I_{h}$, the high dynamic range compression operator $f(*)$ stacks the radiance map in a low dynamic range $I_{l}=f\left(I_{h}\right)$. If image is zoomed into a square window $w_{i}$ having few pixels with ith pixel within center, for this the linear function

$$
I^{l}(j)=p_{i} I^{h}(j)+q_{i}, \quad j \epsilon w_{i} \quad \ldots
$$

Provides an uncomplicated representation which satisfy the local monotonic constraint. where $\mathrm{p}_{\mathrm{i}}, \mathrm{q}_{\mathrm{i}}$ are coefficients within the local window which control the mapping process in two approaches. The value of $q$ impels the base radiance level while $\mathrm{p}$, determines the slope of linear function.

Adding all the local linear equations as defined for individual windows, an image may be reconstructed by minimizing the following equation

$$
\sum_{i} \sum_{j \epsilon w_{i}}\left(I^{l}(j)-p_{i} I^{h}(j)-q_{i}\right)^{2}
$$

where $i$ adds over all pixels in the HDR image. As $\mathrm{P}_{\mathrm{i}}$ straight away controls local contrast, we may express final objective function optimally minimized as (given the large set of unknowns)

$f=\sum_{i}\left(\sum_{j \in w_{i}}\left(I^{l}(j)-p_{i} I^{h}(j)-q_{i}\right)^{2}+\epsilon c_{i}\left(p_{i}-c_{i}\right)^{2}\right)$

where $c_{i}$ is a positive pre-set value to guide the modification of local contrast. However, each pair of linear coefficients $[p i, q i]$ is defined only for a single window, minimization of eq (3) may be rewritten as

$$
\begin{aligned}
& \underset{p, q_{1} I^{T^{*}}}{\arg \min } f=\underset{I^{1}}{\arg \min } \sum_{i} \underset{p t, q_{i}}{\arg \min } f_{i}, \quad \text { where } \\
& f_{\mathrm{f}}=\left(\sum_{j \in w_{i}}\left(I^{l}(j)-p_{i} I^{h}(j)-q_{t}\right)^{2}+E c_{t}^{-2}\left(p_{t}-c_{i}\right)^{2}\right) .
\end{aligned}
$$

Using the above expression, we first compute the optimal solution of $\left(\mathrm{p}_{\mathrm{i}}, \mathrm{q}_{\mathrm{i}}\right)$. 
RGB channels are reconstructed after obtaining the tone mapped radiance map $I_{l}$ using [12]

$I_{k}^{l}(i)=\left(\frac{I_{k}^{h}(i)}{I^{h}(i)}\right)^{s} \times I^{l}(i), \quad k \in\{r, g, b\} \ldots$

where $I_{K}^{l}$ is one RGB color channel in the given image, $I_{K}^{h}$ is the color channel in the input HDR image and $S$ is the saturation factor.

\section{M-ESTIMATION BASED IMAGE ENHANCEMENT AND SYNTHESIS}

Eq (4) may be minimized using M-estimation method, Let $I_{i}$ be the $i t h$ error data of the variation between ith observation and the fitted value. The $M$-estimators try to minimize the impact of outliers by substituting the squared residuals, $I_{i}^{2}$, by slowly increasing function $\rho$, yielding

where $\rho$ is penalty function with a minimum at zero and opted to be less than square. If $\rho$ has a derivative $\left(\psi=\rho^{\prime}\right.$, called influence fuction), then the solution to (6) elates the following equation

$$
\sum_{i=1}^{N} \psi\left(I_{i}-p_{i} I^{h}(i)-q_{i}\right) I_{i}^{l}=0, l=1 \ldots L
$$

An estimator defined in (6) or (7) as an M-estimator. Hampel estimator [10] is used for minimization. Skeleton of proposed tone mapping algorithm is presented in Table.

\begin{tabular}{|l}
$\begin{array}{l}\text { 1. Input: An HDR image with radiance map } I^{n} \text {. } \\
\text { 2. Main Steps: } \\
2.1 \quad \text { Generate the guidance map } c \text { according to (6) } \\
\text { in Section 4. } \\
2.2 \text { Construct matrix } S \text { and } B \text { according to (18) in } \\
\text { the Appendix. } \\
2.3 \text { Compute } I^{l} \text { by solving the linear system de- } \\
\text { fined in (17) in the Appendix. } \\
2.3 \text { Restore the RGB channels } I_{k}^{l} \text { in the tone } \\
\text { mapped result by (5). }\end{array}$ \\
3. Output: An ordinary image with color channels $I_{k}^{l}$
\end{tabular}

\section{TABLE 1}

Skeleton of our tone mapping algorithm.

\section{SIMULATION RESULTS}

From the following simulation results shown in figure, it is observed that the high definition range image (memorial church image) tone mapping results are shown in figure. Radiance of dark regions of the given image, for a specification, the upper left corner of image is raise for low to high. The brightness of the smudged glass of window is minimized, revealing more clear structural details. The paintings and textures on the ceiling and wall are also clearly preserved. These simulation results are described with the parameters $\beta_{1}$ from 0.4 to 1.3 with an increment of $0.05, \beta_{2}$ as 0 and $\beta_{3}$ as 0.1 . It can also be depicted that the high definition range is compressed with linearly increased ratio and more bright and dark regions details are maintained using larger $\beta_{1}$.

$$
\underline{\theta}=\underset{\underline{\theta} \in \Re^{K}}{\arg \min } \sum_{i=1}^{N} \rho\left(\left(I_{i}-p_{i} I^{h}(i)-q_{i}\right)^{2}+\varepsilon c_{i}^{2}\left(p_{i}-c_{i}^{2}\right)\right)
$$
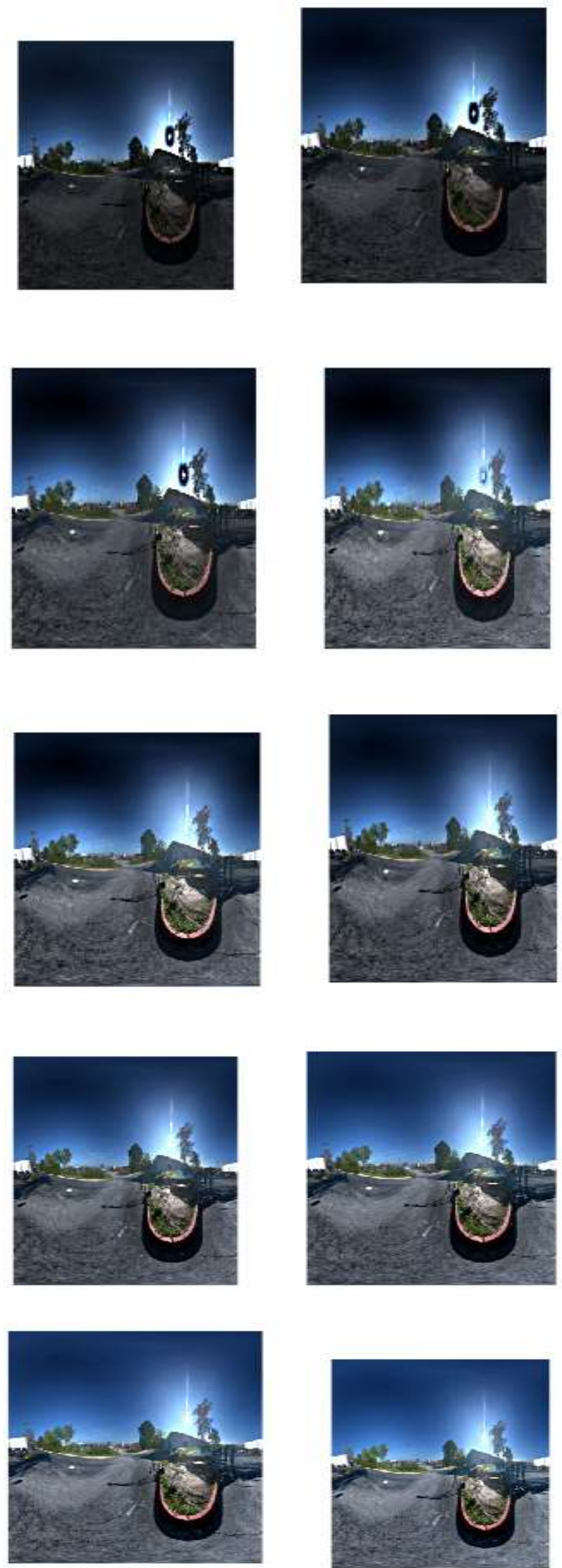

Published By: 


\section{CONCLUDING REMARKS}

An optimal HDR image compression method which is used to suppress the global contrast without losing details of local image structure is proposed in this paper. The proposed technique uses m-estimation based non-linear method for image radiance reconstruction. The proposed method may also be applied for LDR image enhancement and expansion using the same framework. Further, in the proposed method parameter adjustment is simple. Local radiance may be modified using Stroke brushes to specify the preferred values. The Efficiency of the proposed method is demonstrated using simulation results as discussed in the paper.

\section{REFERENCES}

1. K. Devlin, "A review of tone reproduction techniques," Technical Report CSTR-02-005, 2002.

2. E. Reinhard, G. Ward, S. Pattanaik, and P. Debevec. High Dynamic Range Imaging. Morgan Kaufman, 2005.

3. Z. Farbman, R. Fattal, D. Lischinski, and R. Szeliski, "Edge preserving decompositions for multi-scale tone and detail manipulation," In SIGGRAPH, 2008.

4. N. Neelima and Y Ravi Kumar, "Optimal clustering based outlier detection and cluster center initialization algorithm for effective tone mapping," Multimedia Tools and Applications, 2019.

5. P. J. Huber, Robust Statistics. New York: Wiley, 1981.

6. F. R. Hampel et al., Robust Statistics: The Approach Based on Influence Functions. New York: Wiley, 1986. 